\title{
Noncommutative Root Space Witt, Ricci Flow, and Poisson Bracket Continual Lie Algebras
}

\author{
Alexander ZUEVSKY \\ School of Mathematics, Statistics and Applied Mathematics, National University of Ireland, \\ Galway, Ireland \\ Max-Planck Institut für Mathematik, Vivatsgasse 7, 53111, Bonn, Germany \\ E-mail: zuevsky@mpim-bonn.mpg.de
}

Received August 21, 2009, in final form November 16, 2009; Published online November 19, 2009 doi:10.3842/SIGMA.2009.105

\begin{abstract}
We introduce new examples of mappings defining noncommutative root space generalizations for the Witt, Ricci flow, and Poisson bracket continual Lie algebras.
\end{abstract}

Key words: continual Lie algebras; noncommutative integrable models

2000 Mathematics Subject Classification: 35Q58; 37K05; 37K30

\section{Introduction}

The notion of a continual Lie algebra (a Lie algebra with continual set of roots) first appeared in works of Saveliev and Vershik [17, 18, 19, 12]. The main feature which distinguishes continual Lie algebras from ordinary ones is that their generators are parametrized by elements of a space which we call in what follows the root space $\mathcal{E}$. The commutator of two continual Lie algebra elements also depends on bilinear mappings $K$ defined on the root space. Continual Lie algebras [18] represent generalizations of many classes of infinite-dimensional Lie algebras. In the simplest class of continual Lie algebra examples, Kac-Moody Lie algebras [11], mappings $K$ reduce to Cartan matrices corresponding to discrete root spaces.

The general definition of a continual Lie algebra $[18,19]$ admits a noncommutative space of roots. The identities which follow from Jacobi identity applied to elements of a continual Lie algebra are also valid in noncommutative case. Nevertheless, it is quite complicated to find examples (some of them were derived in [23]). Most cases of mappings defining continual Lie algebras with commutative root spaces do not satisfy to these identities when one passes to a noncommutative space. It is even more complicated when original mappings contain derivative terms. Our aim was to find classes of appropriate differential mappings that are subject to the identities (5)-(8) when $\mathcal{E}$ is noncommutative.

In this paper we introduce generalizations for the Witt [6], Ricci flow [2, 3, 4, 24], and Poisson bracket [18], as well as other examples of continual Lie algebras with noncommutative root spaces. The noncommutative root space $\mathcal{E}$ we use is the space of tensor product powers of a space $E$ which is endowed with a noncommutative product. We also introduce some special products defined in $\mathcal{E}$. The associativity of these products leads to solutions of the identities (5)-(8). We would like to stress that we derive mappings for new continual Lie algebras independently of the nature of a noncommutative product in $E$. In further applications one can then consider examples of continual Lie algebras with some specific multiplications in $E$ (as in, e.g., see [7]), and with some fixed commutation relations of $E$ elements and their derivatives with respect to parameters. New examples of continual Lie algebras we introduce generalize corresponding commutative root space counterparts. In the special limit of tensor product power one and commutative limit of a product in $E$, they reduce to original continual Lie algebras. 
In some cases mappings of noncommutative root space continual Lie algebras we derive do not form continual Lie algebras in commutative limit.

Many interesting properties of continual Lie algebras have been found useful in applications within the group-theoretical approach [13] to the construction of exactly solvable dynamical systems $[18,19,2,3,4,23,22]$. The structure of commutations relations and bilinear mappings $K$ turned out to be very helpful in the development of noncommutative generalizations for known integrable models [23, 22, 24]. Continual Lie algebras with noncommutative root space we introduce inspire various new applications in integrable models. In particular, they can be used (as in [23]) for construction of solvable models defined in noncommutative spaces [21, 8, 22].

The plan of the paper is as follows. In Section 2 we give a formal definition of a continual Lie algebra. Then in Section 3 examples of continual Lie algebras with commutative root spaces are mentioned. In Section 4 we give definitions and notations related to noncommutative spaces of roots (NCRS). In Section 5 we introduce new examples of mappings in a noncommutative root space $\mathcal{E}$, and prove that they satisfy to the defining identities of a continual Lie algebra. Finally, we mention directions for further generalizations and possible applications in solvable models.

\section{Continual Lie algebras}

Let $\mathcal{E}$ be a vector space. A continual Lie algebra [12] is generated by the generalized local part $\mathcal{G}^{m_{0}}=\oplus_{|n| \leq m_{0}} \mathcal{G}_{n}, \mathcal{G}_{n}=\left\{X_{n}(\phi), \phi \in \mathcal{E}\right\}, n \in \mathbb{Z}$, satisfying the defining relations for all $\phi, \psi \in \mathcal{E}$, and $|n|,|m|,|n+m| \leq m_{0}$,

$$
\left[X_{n}(\phi), X_{m}(\psi)\right]=X_{n+m}\left(K_{n, m}(\phi, \psi)\right),
$$

where $K_{n, m}: \mathcal{E} \times \mathcal{E} \rightarrow \mathcal{E}, n, m \in \mathbb{Z}$, are bilinear mappings. As for classical discrete root space Lie algebras, we call $\mathcal{E}$ the root space. Ordinary Jacobi identity applied to elements $X_{i}(\phi)$ imply the following conditions on $K_{n, m}$ :

$$
\begin{aligned}
& K_{k, m+n}\left(\phi, K_{m, n}(\psi, \chi)\right)+K_{m, n+k}\left(\psi, K_{n, k}(\chi, \phi)\right)+K_{n, k+m}\left(\chi, K_{k, m}(\phi, \psi)\right)=0, \\
& K_{n, m}(\phi, \psi)=-K_{m, n}(\psi, \phi),
\end{aligned}
$$

for all $\phi, \psi, \chi \in \mathcal{E}$, and $|l| \leq m_{0}$, where $l$ denotes an index (or a sum of indexes) in (2). Then an infinite-dimensional algebra $\mathcal{G}(\mathcal{E} ; K)=\mathcal{G}^{\prime}(\mathcal{E} ; K) / J$ is called a continual contragredient Lie algebra where $\mathcal{G}^{\prime}(\mathcal{E} ; K)$ is a Lie algebra freely generated by the minimal (in accordance with $m_{0}$ ) generalized local part $\mathcal{G}^{m_{0}}$, and $J$ is the largest homogeneous ideal with trivial intersection with $\mathcal{G}_{0}$ (consideration of the quotient is equivalent to imposing the Serre relations in ordinary Lie algebra case) $[18,19]$. When $\left|m_{0}\right| \leq 1$, the commutation relations (1) have the form

$$
\begin{aligned}
& {\left[X_{0}(\phi), X_{0}(\psi)\right]=X_{0}\left(K_{0,0}(\phi, \psi)\right), \quad\left[X_{0}(\phi), X_{ \pm}(\psi)\right]=X_{ \pm}\left(K_{ \pm}(\phi, \psi)\right),} \\
& {\left[X_{+}(\phi), X_{-}(\psi)\right]=X_{0}\left(K_{0}(\phi, \psi)\right),}
\end{aligned}
$$

for all $\phi, \psi \in \mathcal{E}$, and the conditions (2), (3) reduce to

$$
\begin{aligned}
& K_{0,0}(\phi, \psi)=-K_{0,0}(\psi, \phi), \\
& K_{ \pm}\left(K_{0,0}(\phi, \psi), \chi\right)=K_{ \pm}\left(\phi, K_{ \pm}(\psi, \chi)\right)-K_{ \pm}\left(\psi, K_{ \pm}(\phi, \chi)\right), \\
& K_{0,0}\left(\psi, K_{0}(\phi, \chi)\right)=K_{0}\left(K_{+}(\psi, \phi), \chi\right)+K_{0}\left(\phi, K_{-}(\psi, \chi)\right), \\
& K_{0,0}\left(\phi, K_{0,0}(\psi, \chi)\right)+K_{0,0}\left(\psi, K_{0,0}(\chi, \phi)\right)+K_{0,0}\left(\chi, K_{0,0}(\phi, \psi)\right)=0 .
\end{aligned}
$$

\section{Examples of continual Lie algebras}

In this section $\mathcal{E}$ is a space of complex differentiable functions. Here we give examples of continual Lie algebras with commutative $\mathcal{E}$, relevant to the constructions of this paper. Other examples can be found in $[12,17,18,19]$. 


\subsection{Witt algebra}

The Witt algebra [6] is the centerless Virasoro algebra. The commutation relation on the single generator $X(\phi)$, for $\phi, \psi \in \mathcal{E}$, are

$$
\begin{aligned}
& {[X(\phi), X(\psi)]=X(\phi \partial \psi-\psi \partial \phi) \equiv X\left(\left[\phi,{ }^{\partial} \psi\right]\right),} \\
& K(\phi, \psi)=\phi \partial \psi-\psi \partial \phi,
\end{aligned}
$$

where $\partial$ denotes the differentiation with respect to a real parameter with obvious notation after the last equality in (9). The only condition that the mapping $K(\phi, \psi)$ satisfies is (2) with $k=m=n=0$.

\subsection{Ricci flow algebra}

The Ricci flow algebra $[2,3,4]$ is determined by the bilinear mappings

$$
K_{0,0}(\phi, \psi)=0, \quad K_{ \pm}(\phi, \psi)=\mp \phi \cdot \psi, \quad K_{0}(\phi, \psi)=\partial(\phi \cdot \psi) .
$$

The set of the mappings (11) is equivalent to the set $K_{0}(\phi, \psi)=\phi \cdot \psi, K_{ \pm}(\phi, \psi)=\mp \phi \cdot \partial \psi$, $K_{0,0}(\phi, \psi)=0$. It is easy to see that both sets obey the conditions (5)-(8). The bicomplex construction $[4,24]$ based on generators of this Lie algebra leads to the simplest example of the Ricci flow equation.

\subsection{Poisson bracket algebra}

The third example we consider in this section is a continual Lie algebra defined by the mappings

$$
K_{0,0}(\phi, \psi)=0, \quad K_{ \pm}(\phi, \psi)=\mp i \partial \phi \cdot \psi, \quad K_{0}(\phi, \psi)=-i \partial(\phi \cdot \psi),
$$

and $K_{n, m}(\phi, \psi)=i(n \partial \psi \cdot \phi-m \partial \phi \cdot \psi), n, m \in \mathbb{Z}$. In [18] it was proved that this continual Lie algebra is isomorphic to the Poisson bracket algebra under an appropriate substitution of variables.

\section{Noncommutative tensor product root space}

In all examples of continual Lie algebras mentioned above $\mathcal{E}$ is necessarily a commutative space. This requirement is dictated by the identities (5)-(8). Indeed, to prove (5)-(8) by substituting corresponding mappings one should be able to interchange elements of $\mathcal{E}$. Certain problems could also be caused by the presence of derivatives in mappings. Note again that the general definition of a continual Lie algebra admits a noncommutative space of roots, although almost all examples of continual Lie algebras do not survive in noncommutativity. In order to overcome these difficulties we define continual Lie algebras with mappings acting in the space of tensor product powers of a noncommutative space $E$ [1]. We introduce counterparts of continual Lie algebras discussed in previous section by defining new mappings while keeping the form of commutation relations (4). Since the relations (5)-(8) for a continual Lie algebra mappings come from ordinary Jacobi identity they are preserved in the root space we use.

First we give some definitions and notations. Let $E$ be a noncommutative algebra with a product . Then let $\mathcal{E}$ be the space of all tensor powers of $E$ (including possibly infinite or semi-infinite powers). For simplicity we take identical copies of $E$ in $\mathcal{E}$, though a generalization with nonidentical $E$ is also possible. We denote by $E^{\otimes^{M}}=E \otimes \cdots \otimes E$, subspaces of $\mathcal{E}$ containing $M$ copies of $E$. For a monome $\phi \in \mathcal{E}$, which belongs to a subspace $E^{\otimes^{N}}$ we define (possibly infinite) ord $\phi=N$. The product of two elements $\phi, \psi$, ord $\phi=\operatorname{ord} \psi$, in the tensor 
algebra $\mathcal{E}$ is defined standardly (we skip the sign of the product) $\phi \psi=\bigotimes_{i=1}^{\text {ord } \phi} \phi_{i} \cdot \psi_{i}$, where $\bigotimes_{n} \eta_{n}$, denotes the ordered tensor product.

Let us introduce two new operations. The "gluing" (or concatenation) operation $`$ for two finite order elements $\phi, \psi \in \mathcal{E}$ :

$$
\begin{aligned}
\widehat{\phi} \psi & =\phi_{1} \otimes \cdots \otimes\left(\phi_{\operatorname{ord} \phi} \cdot \psi_{1}\right) \otimes \cdots \otimes \psi_{\operatorname{ord} \psi} \\
& =\left(\bigotimes_{n=1}^{\operatorname{ord} \phi-1} \phi_{n}\right) \otimes\left(\phi_{\operatorname{ord} \phi} \cdot \psi_{1}\right) \otimes\left(\bigotimes_{m=2}^{\operatorname{ord} \psi} \psi_{m}\right) .
\end{aligned}
$$

The gluing operation is a mapping $E^{\otimes^{\text {ord } \phi}} \times E^{\otimes^{\text {ord } \psi}} \longrightarrow E^{\otimes^{\text {ord } \phi+o r d} \psi-1}$. The definition of the gluing operation can be generalized for the case of semi-infinite tensor product elements. One can concatenate left semi-infinite with right semi-infinite elements.

Suppose $E$ possesses also a formal derivative operation $\partial$ (e.g., with respect to a real parameter) parameter. In some cases we also assume the existence of the inverse (with respect to a product defined in $E$ ) operator $\partial^{-1}$ to the formal derivative $\partial$. Then for $c \in E$, we define a differential operator $D_{k}, 1 \leq k \leq \operatorname{ord} \phi$, which acts on the ord $\phi$ tensor power element $\phi \in \mathcal{E}$, as follows

$$
D_{k} \phi=\phi_{1} \otimes \cdots \otimes c \cdot \partial \phi_{k} \otimes \cdots \otimes \phi_{\text {ord } \phi}=\bigotimes_{i=1}^{\operatorname{ord} \phi}(c \cdot \partial)^{\delta_{i, k}} \cdot \phi_{i} .
$$

The gluing operation (13), as well as the action of $D_{\mathrm{ord} \phi}(\hat{\phi} \psi)$, are associative with respect to the tensor product

$$
\begin{aligned}
& \widehat{\phi}(\psi \otimes \chi)=(\widehat{\psi} \widehat{\psi}) \otimes \chi, \\
& D_{\operatorname{ord} \phi}(\widehat{\phi}(\psi \otimes \chi))=\left(D_{\operatorname{ord} \phi}(\widehat{\phi} \psi)\right) \otimes \chi .
\end{aligned}
$$

In what follows an element $c \in E$ is skipped for the sake of simplicity.

We introduce also the following operator in $\mathcal{E}$

$$
\partial^{\otimes \text { ord }} \cdot \phi=(\partial \otimes \cdots \otimes \partial) \cdot \phi=\partial^{\otimes^{\text {ord } \phi}} \cdot \phi,
$$

with the number of derivatives $\partial$ in the tensor product equal to the tensor power order of an element $\mathcal{E}$ which $\partial^{\otimes \text { ord }}$ acts on, e.g., ord $\phi$. We will use two notations for the action of the differentiation on an element $\phi \in \mathcal{E}$,

$$
\partial^{\otimes \text { ord }} \cdot \phi \equiv \phi \cdot \partial^{\otimes \text { ord }}
$$

The natural property of the operator $\partial^{\otimes \text { ord }}$. is obvious

$$
\partial^{\otimes \text { ord }} \cdot(a \otimes b)=\left(\partial^{\otimes \text { ord }} \cdot a\right) \otimes\left(\partial^{\otimes \text { ord }} \cdot b\right),
$$

where here and what follows the --multiplication has higher priority with respect to the tensor product so that we will skip corresponding brackets.

In (9) and what follows we denote by $\left[\phi,{ }^{A} \psi\right]$ the commutator

$$
\left[\phi,{ }^{A} \psi\right]=\phi A \psi-\psi A \phi
$$

where $A$ is an operation inserted in between $\phi$ and $\psi$. We will also use the operator $P_{\otimes}$ which inverts the order of tensor multipliers in an element of $\mathcal{E}$. Note that for $\phi, \psi \in \mathcal{E}$,

$$
P_{\otimes}(\phi \otimes \psi)=P_{\otimes}(\psi) \otimes P_{\otimes}(\phi) .
$$


Having defined mappings for new continual Lie algebras with noncommutative spaces of roots $\mathcal{E}$ we have to establish connections with their commutative counterparts, i.e., corresponding continual Lie algebras with commutative root spaces. In order to do so we consider the following commutative limit. Firstly, we reduce $\mathcal{E}$ to $E$ by taking the tensor order of $\mathcal{E}$ equal to one. All tensor power product operations present in defining continual Lie algebra mappings $K$ have to be also replaced with corresponding tensor product power one operations acting in $E$. Then we pass to a commutative ordinary product limit in all actions of operators in $E$ involving a noncommutative $E$ product. Alternatively one can consider another limit when all tensor products have to be replaced by a noncommutative product in $E$, and then pass to its commutative limit. In some cases in our constructions we do not assume that formal derivative operations obey Leibniz rule with respect to a noncommutative product in $E$ or tensor product in $\mathcal{E}$. In the rest of the paper we proceed with examples of new continual Lie algebras with noncommutative root spaces $(\mathrm{NCRS}) \mathcal{E}$.

\section{New examples of NCRS continual algebras}

\section{$5.1 \quad$ NCRS Witt algebra}

We start with the most trivial case. One can guess the following mapping

$$
\begin{aligned}
& K(\phi, \psi)=\phi^{\widehat{D}} D_{1} \psi-\widehat{\psi} D_{1} \phi \\
& =\phi_{1} \otimes \cdots \otimes\left(\phi_{\text {ord } \phi} \cdot \partial \psi_{1}\right) \otimes \cdots \otimes \psi_{\text {ord } \phi}-\psi_{1} \otimes \cdots \otimes\left(\psi_{\text {ord } \psi} \cdot \partial \phi_{1}\right) \otimes \cdots \otimes \phi_{\text {ord } \phi} \\
& =\left[\phi,{ }^{\wedge} \cdot D_{1} \psi\right] \text {. }
\end{aligned}
$$

We call a continual Lie algebra defined by (17) the noncommutative root space Witt continual Lie algebra.

Remark 1. When ord $\phi=$ ord $\psi=1$ in (17), the mapping formally coinsides with (10), though the proof is still valid since (2) has a different sense (see (34)).

\subsection{NCRS Ricci flow algebra}

Following the idea given in previous subsection we introduce the following mappings $K_{0}, K_{ \pm}$, $K_{0,0}$ :

$$
\begin{aligned}
& K_{+}(\phi, \psi)=\phi \otimes \psi, \\
& K_{-}(\phi, \psi)=-\psi \otimes \phi, \\
& K_{0,0}(\phi, \psi)=\left[\phi,{ }^{\otimes} \psi\right], \\
& K_{0}(\phi, \psi)=D_{\text {ord } \phi}\left(\phi^{\widehat{\psi}} \psi\right),
\end{aligned}
$$

where

$$
D_{\text {ord } \phi}(\widehat{\phi} \psi)=\phi_{1} \otimes \cdots \otimes \partial\left(\phi_{\text {ord } \phi} \cdot \psi_{1}\right) \otimes \cdots \otimes \psi_{\text {ord } \psi}
$$

We call a continual Lie algebra defined by the mappings (18)-(21) the noncommutative root space Ricci flow continual Lie algebra. The relation (5) is hold in (ord $\phi+\operatorname{ord} \psi$ )-tensor power of $E,(6),(8)$ are hold in (ord $\phi+\operatorname{ord} \psi+\operatorname{ord} \chi)$-power, while the relation (7) is in (ord $\phi+$ ord $\psi+$ ord $\chi-1)$. In the commutative limit, we put ord $\mathcal{E}=1$, substitute all tensor product remaining in mappings by the product in $E$, and then consider $E$ being commutative. Then we see that the mappings (18)-(21) have the commutative limit (11). 


\subsection{NCRS Poisson bracket algebra}

The case of the Poisson bracket algebras seems to be more complicated. We introduce a continual Lie algebra over a noncommutative root space so that its mappings satisfy to the identities (5)-(8), and comply with a commutative limit (12). Consider

$$
\begin{aligned}
& K_{+}(\phi, \psi)=-i \partial^{\otimes \text { ord }} \cdot \phi \otimes \psi, \\
& K_{-}(\phi, \psi)=i \psi \otimes \partial^{\otimes \text { ord }} \cdot \phi, \\
& K_{0,0}(\phi, \psi)=-i\left[\phi,{ }^{\otimes} \psi\right], \\
& K_{0}(\phi, \psi)=-i\left(\partial^{-1}\right)^{\otimes \text { ord }} \cdot D_{\text {ord } \phi}^{2} \cdot\left(\phi^{-} \psi\right),
\end{aligned}
$$

where the operator $\left(\partial^{-1}\right)^{\otimes \text { ord }} \cdot$ acts similar to $(14)$.

Remark 2. Note that $K_{ \pm}, K_{0,0}(22)-(25)$ have the Poisson bracket continual Lie algebra mappings as a commutative limit. As specified above, we put ord $\mathcal{E}=1$, substitute all tensor product remaining in mappings by the product in $E$, and then consider a commutative limit of the product in $E$. Then $K_{0,0}(\phi, \psi)=0$, and $K_{0}(\phi, \psi)=-i \partial(\phi \cdot \psi), K_{ \pm}(\phi, \psi)=\mp i \partial \phi \cdot \psi$.

Remark 3. The mappings defining the higher grading subspaces of the Ricci flow continual Lie algebra (11), as well as for the Ricci flow (18)-(21) and Poisson bracket (22)-(25) continual Lie algebras with noncommutative root spaces will be discussed in a separate paper.

\subsection{Further examples}

In this subsection we give further examples of noncommutative root space continual Lie algebras whose mappings do not form continual Lie algebras with commutative root spaces in the commutative limit.

\subsubsection{NCRS Poisson-type bracket continual Lie algebras}

For $\phi, \psi \in \mathcal{E}$, consider the mappings:

$$
\begin{aligned}
& K_{0}(\phi, \psi)=-i D_{\text {ord } \phi}(\widehat{\phi} \psi) \text {, } \\
& K_{+}(\phi, \psi)=-i \phi^{-}\left(D_{1} \psi\right) \text {, } \\
& K_{-}(\phi, \psi)=i \psi^{-}\left(D_{1} \phi\right) \text {, } \\
& K_{0,0}(\phi, \psi)=K_{+}(\phi, \psi)+K_{-}(\phi, \psi)=-i\left[\phi,{ }^{\cdot} D_{1} \psi\right] .
\end{aligned}
$$

In the commutative limit, ord $\mathcal{E}=1$, and commutative product in $E$, the mappings (26)-(29) reduce to $K_{0}(\phi, \psi)=-i \partial(\phi \psi), K_{+}(\phi, \psi)=-i \phi \partial \psi, K_{-}(\phi, \psi)=i \psi \partial \phi, K_{0,0}(\phi, \psi)=-i\left[\phi,{ }^{\partial} \psi\right]$. It is easy to check that this set of mappings does not satisfy to (5)-(8), and therefore does not define a continual Lie algebra. We see that the form of the mapping $K_{-}(28)$ represents a direct noncommutative analog of the Poisson bracket continual Lie algebra mapping $K_{-}$of (12) with the commutative root space, while $K_{+}$-mapping (27) contains the derivative action on the second argument in contrast to $K_{+}$of (12).

Remark 4. One can alternatively define a continual Lie algebra described above by the mappings:

$$
\begin{aligned}
& K_{0}(\phi, \psi)=-i / 2\left(D_{\text {ord } \phi}(\hat{\phi} \psi)+D_{\text {ord } \psi}(\widehat{\psi} \phi)\right), \\
& K_{+}(\phi, \psi)=-i\left(D_{\text {ord } \phi} \phi\right) \wedge \psi \text {, } \\
& K_{-}(\phi, \psi)=i\left(D_{\text {ord } \psi} \psi\right)^{\wedge} \phi \text {, }
\end{aligned}
$$




$$
K_{0,0}(\phi, \psi)=K_{+}(\phi, \psi)+K_{-}(\phi, \psi)
$$

that in the commutative limit provide $K_{0}(\phi, \psi)=-i \partial(\phi \psi), K_{+}(\phi, \psi)=-i(\partial \phi) \psi, K_{-}(\phi, \psi)=$ $i(\partial \psi) \phi, K_{0,0}(\phi, \psi)=i((\partial \psi) \phi-(\partial \phi) \psi)$, and, as in (26)-(29), do not correspond a continual Lie algebra with a commutative root space. We call these two examples the Poisson-type bracket continual Lie algebras.

\subsubsection{NCRS Integral mapping continual Lie algebra}

Let $E$ be the ring of differentiable functions with a noncommutative product - and differentiation $\partial$. Then let $\mathcal{E}$ be the algebra of tensor powers of $E$. We then introduce the mappings of a new continual Lie algebra with noncommutative root space $\mathcal{E}$ and the mapping $K_{0}$ of integral type

$$
\begin{aligned}
& K_{+}(\phi, \psi)=-i\left[\phi \cdot \partial^{\otimes \text { ord }} \otimes \psi+\psi \otimes \partial^{\otimes \text { ord }} \cdot P_{\otimes} \phi\right], \\
& K_{-}(\phi, \psi)=i\left[\left(P_{\otimes} \phi\right) \cdot \partial^{\otimes \text { ord }} \otimes \psi+\psi \otimes \partial^{\otimes \text { ord }} \cdot \phi\right], \\
& K_{0,0}(\phi, \psi)=-i\left[\phi,{ }^{\otimes} \psi\right], \\
& K_{0}(\phi, \psi)=-i\left(\partial^{-1}\right)^{\otimes \text { ord }} \cdot(\phi \otimes \psi) .
\end{aligned}
$$

The terms containing $P_{\otimes}$-operator in (30) and (31) are not important for the construction of corresponding continual Lie algebra (see the proof below) and are not present in commutative case. It is a usual situation since noncommutative counterparts for derivative terms are not unique in general. In contrast to the case of the noncommutative Poisson bracket continual Lie algebra the derivative acts on the second argument in $K_{+}(\phi, \psi)$.

Remark 5. In the commutative limit the mappings $K_{ \pm}, K_{0,0}(30)-(33)$ reduce to $K_{0,0}(\phi, \psi)=0$, $K_{0}(\phi, \psi)=-i \partial^{-1}(\phi \cdot \psi), K_{ \pm}(\phi, \psi)=\mp i(\phi \cdot \partial \psi+\psi \cdot \partial \phi)$, and do not form a continual Lie algebra.

Remark 6. In a similar fashion, we can introduce noncommutative counterparts for other examples of continual Lie algebras (in particular for vector fields and diffeomorphisms, and cross-product continual Lie algebras [18]) with commutative $\mathcal{E}$ described in [12, 19]. The case of contact Lie algebras $[15,16]$ with noncommutative root spaces will be considered elsewhere.

\subsection{Main statement}

Now we will show that new examples of mappings acting on noncommutative root spaces in subsections above do indeed comply with the definition of a continual Lie algebra.

Proposition 1. The mappings a) (17), b) (18)-(21), c) (22)-(25), d) (26)-(29), e) (30)-(33) satisfy to the identities (5)-(8) and define noncommutative root space continual Lie algebras.

The cases $a), b), c$ ) represent generalizations for the Witt, Ricci flow, and Poisson bracket continual Lie algebras correspondingly.

Proof. a) The relation (5) is satisfied trivially. For (8) one has

$$
\begin{aligned}
& K(\phi, K(\psi, \chi))+K(\psi, K(\chi, \phi))+K(\chi, K(\phi, \psi))
\end{aligned}
$$

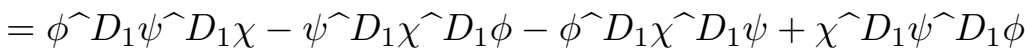

$$
\begin{aligned}
& + \text { two permutations of }(\phi, \psi, \chi)=0 \text {. }
\end{aligned}
$$

Then we see that all terms in the above expression cancel. 
b) (5) and (8) trivially follow from the definition (20) of $K_{0,0}$. The identity (6) follows from the definitions of $K_{0,0}(20)$ and $K_{ \pm}(18)-(19)$. For (7) one has for any $\phi, \psi, \chi \in \mathcal{E}$,

$$
\begin{aligned}
& K_{0,0}\left(\psi, K_{0}(\phi, \chi)\right)=\left[\psi,{ }^{\otimes} \phi_{1} \otimes \cdots \otimes \partial\left(\phi_{\operatorname{ord} \phi} \cdot \chi_{1}\right) \otimes \cdots \otimes \chi_{\operatorname{ord}}\right] \\
&= D_{\text {ord } \phi}\left(\psi \otimes \phi_{1} \otimes \cdots \otimes \phi_{\operatorname{ord} \phi} \cdot \chi_{1} \otimes \cdots \otimes \chi_{\operatorname{ord} \chi}\right) \\
&-D_{\operatorname{ord} \phi}\left(\phi_{1} \otimes \cdots \otimes \phi_{\operatorname{ord} \phi} \cdot \chi_{1} \otimes \cdots \otimes \chi_{\operatorname{ord} \chi} \otimes \psi\right) \\
&= K_{0}(\psi \otimes \phi, \chi)-K_{0}(\phi, \chi \otimes \psi) \\
&= K_{0}\left(K_{+}(\psi, \phi), \chi\right)+K_{0}\left(\phi, K_{-}(\psi, \chi)\right) .
\end{aligned}
$$

c) The identities (5) and (8) are trivially satisfied by $K_{0,0}(24)$. Then we check (6) for (22) and (24):

$$
\begin{aligned}
K_{+}( & \left.\phi, K_{+}(\psi, \chi)\right)-K_{+}\left(\psi, K_{+}(\phi, \chi)\right) \\
& =-\left(\partial^{\otimes \text { ord }} \cdot \phi\right) \otimes\left(\partial^{\otimes \text { ord }} \cdot \psi\right) \otimes \chi+\left(\partial^{\otimes \text { ord }} \cdot \psi\right) \otimes\left(\partial^{\otimes \text { ord }} \cdot \phi\right) \otimes \chi \\
& =-i \partial^{\otimes \text { ord }} \cdot\left(-i\left[\phi,{ }^{\otimes} \psi\right]\right) \otimes \chi \\
& =K_{+}\left(K_{0,0}(\phi, \psi), \chi\right),
\end{aligned}
$$

and similarly for $K_{-}(23)$. Here we have made use of the property (15). Next we check (7)

$$
\begin{aligned}
& K_{0}\left(K_{+}(\psi, \phi), \chi\right)+K_{0}\left(\phi, K_{-}(\psi, \chi)\right)=K_{0}\left(-i \partial^{\otimes \text { ord }} \cdot \psi \otimes \phi, \chi\right)+K_{0}\left(\phi, i \chi \otimes \partial^{\otimes \text { ord }} \cdot \psi\right) \\
& =-\left(\partial^{-1}\right)^{\otimes \text { ord }} \cdot\left[\partial^{\otimes \text { ord }} \cdot \psi \otimes D_{\text {ord } \phi}^{2} \cdot\left(\phi^{\widehat{-} \chi}\right)-D_{\text {ord } \phi}^{2} \cdot\left(\phi^{\widehat{-} \chi}\right) \otimes \partial^{\otimes \text { ord }} \cdot \psi\right] \\
& =-\left[\psi,{ }^{\otimes}\left(\partial^{-1}\right)^{\otimes \text { ord }} \cdot D_{\text {ord } \phi}^{2} \cdot\left(\phi^{\widehat{\chi} \chi}\right)\right] \\
& =K_{0,0}\left(\psi,-i\left(\partial^{-1}\right)^{\otimes \text { ord }} \cdot D_{\text {ord } \phi}^{2} \cdot\left(\phi^{\widehat{\chi} \chi}\right)\right) \\
& =K_{0,0}\left(\psi, K_{0}(\phi, \chi)\right) \text {. }
\end{aligned}
$$

d) As we showed in the proof of $a$ ), (29) satisfy (5) and (8). Then proving (6) one has

$$
\begin{aligned}
K_{+}\left(K_{0,0}(\phi, \psi), \chi\right)= & -\phi_{1} \otimes \cdots \otimes\left(\phi_{\text {ord } \phi} \cdot \partial \psi_{1}\right) \otimes \cdots \otimes\left(\psi_{\operatorname{ord} \psi} \cdot \chi_{1}\right) \otimes \cdots \chi_{\operatorname{ord} \chi} \\
& +\psi_{1} \otimes \cdots \otimes\left(\psi_{\operatorname{ord} \psi} \cdot \partial \phi_{1}\right) \otimes \cdots \otimes\left(\phi_{\operatorname{ord} \phi} \cdot \chi_{1}\right) \otimes \cdots \chi_{\operatorname{ord}} \chi \\
= & K_{+}\left(\phi,-i \psi_{1} \otimes \cdots \otimes\left(\psi_{\operatorname{ord} \psi} \cdot \partial \chi_{1}\right) \otimes \cdots \otimes \chi_{\operatorname{ord} \chi}\right) \\
& -K_{-}\left(\psi,-i \phi_{1} \otimes \cdots \otimes\left(\phi_{\operatorname{ord} \phi} \cdot \partial \chi_{1}\right) \otimes \cdots \otimes \chi_{\operatorname{ord}}\right),
\end{aligned}
$$

and similar for $K_{-}(\phi, \psi)$ in (6). Proving (7) we find

$$
\begin{aligned}
K_{0,0}( & \left.,-i \phi_{1} \otimes \cdots \otimes \partial\left(\phi_{\operatorname{ord} \phi} \cdot \chi_{1}\right) \otimes \cdots \otimes \chi_{\operatorname{ord} \chi}\right) \\
= & -\psi_{1} \otimes \cdots \otimes\left(\psi_{\operatorname{ord} \psi} \cdot \partial \phi_{1}\right) \otimes \cdots \otimes \partial\left(\phi_{\operatorname{ord} \phi} \cdot \chi_{1}\right) \otimes \cdots \otimes \chi_{\text {ord } \chi} \\
& +\phi_{1} \otimes \cdots \otimes \partial\left(\phi_{\text {ord } \phi} \cdot \partial \chi_{1}\right) \otimes \cdots \otimes\left(\chi_{\text {ord } \chi} \cdot \psi_{1}\right) \otimes \cdots \otimes \psi_{\text {ord } \psi} \\
= & K_{0}\left(-i \psi_{1} \otimes \cdots \otimes\left(\psi_{\operatorname{ord} \psi} \cdot \phi_{1}\right) \otimes \cdots, \chi\right) \\
& +K_{0}\left(\phi, i \chi_{1} \otimes \cdots \otimes\left(\chi_{\text {ord } \chi} \cdot \psi_{1}\right) \otimes \cdots \otimes \psi_{\text {ord } \psi}\right) .
\end{aligned}
$$

e) The identities (5) and (8) are trivially satisfied by $K_{0,0}(32)$. We then check (6) for (30) and (33)

$$
\begin{aligned}
K_{+}(\phi, & \left.K_{+}(\psi, \chi)\right)-K_{+}\left(\psi, K_{+}(\phi, \chi)\right) \\
= & -\left(\phi \cdot \partial^{\otimes \text { ord }}\right) \otimes\left(\psi \cdot \partial^{\otimes \text { ord }} \otimes \chi+\chi \otimes \partial^{\otimes \text { ord }} \cdot P_{\otimes} \psi\right) \\
& -\left(\psi \cdot \partial^{\otimes \text { ord }} \otimes \chi+\chi \otimes \partial^{\otimes \text { ord }} \cdot P_{\otimes} \psi\right) \otimes\left(\left(P_{\otimes} \phi\right) \cdot \partial^{\otimes \text { ord }}\right) \\
& +\left(\psi \cdot \partial^{\otimes \text { ord }}\right) \otimes\left(\phi \cdot \partial^{\otimes \text { ord }} \otimes \chi+\chi \otimes \partial^{\otimes \text { ord }} \cdot P_{\otimes} \phi\right)
\end{aligned}
$$




$$
\begin{aligned}
& +\left(\phi \cdot \partial^{\otimes \text { ord }} \otimes \chi+\chi \otimes \partial^{\otimes \text { ord }} \cdot P_{\otimes} \phi\right) \otimes\left(\left(P_{\otimes} \psi\right) \cdot \partial^{\otimes \text { ord }}\right) \\
= & -\left(\phi \cdot \partial^{\otimes \text { ord }} \otimes\left(\psi \cdot \partial^{\otimes \text { ord }}\right)-\psi \cdot \partial^{\otimes \text { ord }} \otimes\left(\phi \cdot \partial^{\otimes \text { ord }}\right)\right) \otimes \chi \\
& -\chi \otimes\left(\left(\partial^{\otimes \text { ord }} \cdot P_{\otimes} \psi\right) \otimes\left(\left(P_{\otimes} \phi\right) \cdot \partial^{\otimes \text { ord }}\right)+\left(\partial^{\otimes \text { ord }} \cdot P_{\otimes} \phi\right) \otimes\left(\left(P_{\otimes} \psi\right) \cdot \partial^{\otimes \text { ord }}\right)\right) \\
= & -i\left(-i\left[\phi,{ }^{\otimes} \psi\right] \cdot \partial^{\otimes \text { ord }} \otimes \chi-i \chi \otimes P_{\otimes} \partial^{\otimes \text { ord }} \cdot\left[\phi,{ }^{\otimes} \psi\right]\right) \\
= & K_{+}\left(K_{0,0}(\phi, \psi), \chi\right),
\end{aligned}
$$

and similarly for the mapping $K_{-}(31)$. Here we have used the properties (15) and (16). We then check the identity (7) for (30)-(33):

$$
\begin{aligned}
K_{0}( & K_{+} \\
= & -\left(\partial^{-1}\right)^{\otimes \text { ord }} \cdot\left(\left[\psi \cdot{ }^{\otimes}\right)+K_{0}\left(\phi, K_{-}(\psi, \chi)\right)\right. \\
& \left.+\left(\partial^{-1}\right)^{\otimes \text { ord }} \cdot\left(\phi \otimes\left[\left(P_{\otimes} \psi\right) \cdot \partial^{\otimes \text { ord }} \otimes \chi+\chi \otimes \partial^{\otimes \text { ord }} \cdot P_{\otimes} \psi\right)\right] \otimes \chi\right) \\
= & -\left(\partial^{-1}\right)^{\otimes \text { ord }} \cdot\left(\psi \cdot \partial^{\otimes \text { ord }} \otimes(\phi \otimes \chi)\right)+\left(\partial^{-1}\right)^{\otimes \text { ord }} \cdot\left((\phi \otimes \chi) \otimes \partial^{\otimes \text { ord }} \cdot \psi\right) \\
= & -\psi \otimes\left(\partial^{-1}\right)^{\otimes \text { ord }} \cdot(\phi \otimes \chi)+\left(\partial^{-1}\right)^{\otimes \text { ord }} \cdot(\phi \otimes \chi) \otimes \psi \\
= & K_{0,0}\left(\psi,-i\left(\partial^{-1}\right)^{\otimes \text { ord }} \cdot(\phi \otimes \chi)\right) \\
= & K_{0,0}\left(\psi, K_{0}(\phi, \chi)\right) .
\end{aligned}
$$

\section{Outlook}

In this paper we construct new examples of continual Lie algebras with noncommutative root spaces. Our considerations are focused on the Witt, Ricci flow, and Poisson bracket continual Lie algebras that contain derivative operations in the defining mappings. When a root space $\mathcal{E}$ is noncommutative, derivative terms cause problems in finding solutions to the identities (5)-(8) following from Jacobi identity. To fix this problem and introduce generalization for the above mentioned continual Lie algebras we have chosen $\mathcal{E}$ to be the space of tensor product powers of a space $E$ with a noncommutative product.

The actions of mappings we use in the space of tensor product powers can be seen as linear part approximations for more general construction of bilinear mappings in $\mathcal{E}$. Even at the this level, the examples of continual Lie algebras introduced in this paper can be generalized. In particular one can use more general mappings containing arbitrary operators $D_{i}, i \in 1, \ldots$, ord $\phi$, $\phi \in \mathcal{E}$, as well as more complicated differential mappings. Relations to [5] as well as interesting generalizations will be discussed elsewhere. We propose to define continual super Lie algebras, $q$-deformations (as in [6]) of the noncommutative continual Witt algebra, and further develop $q$-deformed counterparts [14] for (noncommutative) continual Lie algebras. It would be also interesting to determine possible Hopf algebra structure associated to continual Lie algebras described here. In a noncommutative root space continual Lie algebra construction we use two types of products: a noncommutative product - in a space $E$, and the tensor product defining the algebra $\mathcal{E}$ of tensor powers of $E$. Thus it would be interesting to make connections with [8] where integrable systems have been constructed in spaces with two noncommutative multiplications.

Continual Lie algebras with noncommutative root spaces appear to be attractive objects both from algebraic point of view and in applications in integrable models. In particular, these algebras can define generalizations of certain exactly solvable models. Integrable models defined in noncommutative spaces with ordinary Lie algebras as an algebraic origin were constructed in $[8,9,10,24]$. Using the bicomplex construction [22] for continual Lie algebras with noncommutative root spaces one derives associated dynamical systems in noncommutative spaces. Finally, one can try to define vertex operators [20] as well as vertex operator algebras associated to continual Lie algebras with noncommutative root spaces. 


\section{Acknowledgements}

This work was supported by Science Foundation of Ireland Frontiers of Research Grant. We would like also to thank V. Kac, A. Perelomov, A. Rosenberg, and M. Tuite for valuable discussions.

\section{References}

[1] Atiyah M.F., Macdonald I.G., Introduction to commutative algebra, Addison-Wesley Publishing Co., Reading, Mass. - London - Don Mills, Ont., 1969.

[2] Bakas I., Geometric flows and (some of) their physical applications, hep-th/0511057.

[3] Bakas I., The algebraic structure of geometric flows in two dimensions J. High Energy Phys. 2005 (2005), no. 10, 038, 52 pages, hep-th/0507284.

[4] Bakas I., Ricci flows and their integrability in two dimensions, C. R. Phys. 6 (2005), 175-184, hep-th/0410093.

[5] Berenstein A., Retakh V., Lie algebras and Lie groups over noncommutative rings, Adv. Math. 218 (2008), 1723-1758, math.QA/0701399.

[6] Devchand Ch., Saveliev M.V., Comultiplication for quantum deformations of the centreless Virasoro algebra in the continuum formulation, Phys. Lett. B 258 (1991), 364-368.

[7] Bayen F., Flato M., Fronsdal C., Lichnerowicz A., Sternheimer D., Deformation theory and quantization. I. Deformation of symplectic structures, Ann. Physics 111 (1978), 61-110.

Bayen F., Flato M., Fronsdal C., Lichnerowicz A., Sternheimer D., Deformation theory and quantization. II. Physical applications, Ann. Physics 111 (1978), 111-151.

[8] Dimakis A., Müller-Hoissen F., Bicomplexes and integrable models, J. Phys. A: Math. Gen. 33 (2000), 6579-6591, nlin.SI/0006029.

Dimakis A., Müller-Hoissen F., Bicomplexes, integrable models, and noncommutative geometry, Internat. J. Modern Phys. B 14 (2000), 2455-2460, hep-th/0006005.

[9] Grisaru M.T., Penati S., An integrable noncommutative version of the sine-Gordon system, Nuclear Phys. B 655 (2003), 250-276, hep-th/0112246.

[10] Hamanaka M., Toda K., Towards noncommutative integrable systems, Phys. Lett. A 316 (2003), 77-83, hep-th/0211148.

[11] Kac V.G., Infinite-dimensional Lie algebras, 3rd ed., Cambridge, Cambridge University Press, 1990.

[12] Kashaev R.M., Saveliev M.V., Savelieva S.A., Vershik A.M., On nonlinear equations associated with Lie algebras of diffeomorphism groups of two-dimensional manifolds, in Ideas and Methods in Mathematical Analysis, Stochastics, and Aapplications (Oslo, 1988), Editors S. Albeverio, J.E. Fenstad, H. Holden and T. Lindstrom, Cambridge University Press, Cambridge, 1992, 295-307.

[13] Leznov A.N., Saveliev M.V., Group-theoretical methods for integration of nonlinear dynamical systems, Progress in Physics, Vol. 15, Birkhäuser Verlag, Basel, 1992.

[14] Reshetikhin N.Yu., Semenov-Tian-Shansky M.A., Central extensions of quantum current groups, Lett. Math. Phys. 19 (1990), 133-142.

[15] Saveliev M.V., On an equation associated with the contact Lie algebras, hep-th/9311035.

[16] Saveliev M.V., A nonlinear dynamical system related to the contact Lie algebras, J. Math. Sci. 82 (1996), $3832-3833$.

[17] Saveliev M.V., Integro-differential nonlinear equations and continual Lie algebras, Comm. Math. Phys. 121 (1989), 283-290.

[18] Saveliev M.V., Vershik A.M., Continuum analogues of contragredient Lie algebras (Lie algebras with a Cartan operator and nonlinear dynamical systems), Comm. Math. Phys. 126 (1989), 367-378.

[19] Saveliev M.V., Vershik A.M., New examples of continuum graded Lie algebras, Phys. Lett. A 143 (1990), 121-128.

[20] Saveliev M.V., Zuevsky A.B., Quantum vertex operators for the sine-Gordon model, Internat. J. Modern Phys. A 15 (2000), 3877-3897. 
[21] Seiberg N., Witten E., String theory and noncommutative geometry, J. High Energy Phys. 1999 (1999), no. 09, 032, 93 pages, hep-th/9908142.

Douglas M.R., Nekrasov N.A., Noncommutative field theory, Rev. Modern Phys. 73 (2001), 977-1029, hep-th/0106048.

[22] Zuevsky A., Continual Lie algebra bicomplexes and integrable models, Czechoslovak J. Phys. 55 (2005), $1545-1551$.

[23] Zuevsky A., Continual Lie algebras and noncommutative counterparts of exactly solvable models, J. Phys. A: Math. Gen. 37 (2004), 537-547.

[24] Zuevsky A., Non-commutative Ricci and Calabi flows, Ann. Henri Poincaré 7 (2006), 1569-1578. 